\title{
ARTICLE
}

\section{Gamma dose measurements and spectroscopy analysis for spallation products in JSNS mercury circulation system}

\author{
Yoshimi KASUGAI*, Motoki OOI and Tetsuya KAI \\ Japan Atomic Energy Agency, 2-4, Shirakata Shirane, Tokai-mura, Ibaraki 319-1195, Japan
}

\begin{abstract}
Mercury used as a spallation neutron target at JSNS (J-PARC Spallation Neutron Source) is highly activated by proton beam operation. In order to evaluate the feasibility of hands-on maintenance after nominal beam operation with $1 \mathrm{MW}$ in the hot cell where a mercury circulation system was installed, gamma dose measurements and gamma spectroscopy analysis were carried out after low power operation during the commissioning period. By analyzing the data, it was found that radioactive spallation products adhered to the inner surface of piping wall of the circulation system and those significantly raised the dose rates in the hot cell. In order to discuss about measures for future hot-cell entry, the gamma dose rates after the nominal operation were evaluated based on the measured data obtained in this work.
\end{abstract}

KEYWORDS: J-PARC, MLF, JSNS, Mercury, Spallation Products, Gamma Dose, Gamma-ray Spectroscopy

\section{Introduction}

1. JSNS: J-PARC Spallation Neutron Source.

Materials and Life Science Experimental Facility (MLF) in J-PARC ${ }^{1)}$ is a multipurpose research facility with muon and neutron generation target. The operation of the J-PARC spallation neutron source (JSNS) in MLF started in May 2008 , and an effort is being made now to increase the proton beam intensity up to $1 \mathrm{MW}$ together with many kinds of researches on materials and life science.

\section{Mercury Target and Circulation System.}

In JSNS, mercury was selected as a material of neutron generation target in consideration of the neutron generation efficiency and the cooling. A drawing of the mercury target vessel and a mercury circulation system on a trolley is shown in Fig 1. Neutrons are generated by bombarding mercury in the target vessel with a proton beam, and the mercury heated by incident protons and accompanying nuclear reactions is circulated in the loop between the target vessel and the mercury circulation system for cooling. The target vessel is inserted to the core of the neutron station consisted of moderators, reflector, neutron beam shutters and shields. For the maintenance, the trolley is moved to the "hot cell" prepared for handling of activated devices.

Mercury in the circulation system is highly activated due to radioactive isotopes produced via spallation reactions. Therefore most of the maintenance works for the mercury target and the circulation system were designed to be done by using remote handling system equipped in the cell. However a few works, which is expected to happen once or twice during the facility lifetime of about 30 years, will need to be done by hands-on; this will necessitate entrance of workers to the hot cell.

Mercury in the circulation system can be transferred to the drain tanks under the circulation system on the bottom of the

*Corresponding Author, E-mail: kasugai.yoshimi@jaea.go.jp

(C) Atomic Energy Society of Japan

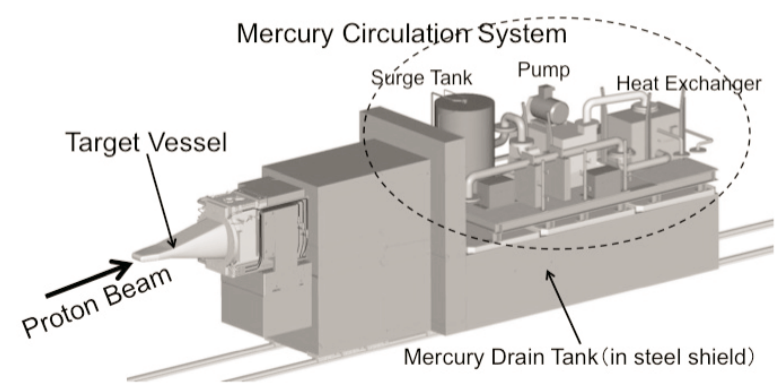

Fig. 1 Mercury target and mercury circulation system on the trolley at JSNS. Mercury in the target vessel is bombarded with protons with energy of $3 \mathrm{GeV}$, and the mercury is circulated in the circulation system for cooling.

trolley (See Fig. 1). The drain tanks are surrounded by a steel shield. Therefore it is expected that the dose rates for external exposure in the hot cell can be reduced by draining the mercury to the drain tanks.

\section{Gamma Dose Estimation behind the mercury} circulation system

In order to evaluate risk of external exposure for the hot-cell entrance after mercury drain, the distribution of gamma dose in the cell was estimated ${ }^{2)}$ under the conditions as follows:

- The proton beam operations are carried out with nominal power and time, $1 \mathrm{MW}$ and $5000 \mathrm{~h} / \mathrm{year}$, for 30 years.

- Cooling for 10 days is performed after termination of the beam operation.

- Induced radioactive isotopes are homogeneously mixed in the mercury.

- Residual mercury exists on the inner surface of the circulation piping and in the components of the circulation system, such as a heat exchanger, valves and a pump; the total weight of the residual was estimated to be about $3 \mathrm{~kg}$.

The evaluation showed that the dose rate at a distance of $10 \mathrm{~m}$ from the back-end of the target trolley was $100 \mu \mathrm{Sv} / \mathrm{h}$, 
which indicated that the hot-cell entry is possible even after the nominal operation. In addition, the evaluation showed that the radioactive isotope of ${ }^{203} \mathrm{Hg}\left(T_{1 / 2}=46.6 \mathrm{~d}\right)$ mainly contributed to the dose in the cell.

\section{Finding an Anomalous Phenomenon}

Since the dose evaluation included large ambiguity about the weight estimation of residual mercury, a test measurement of dose rates just around the circulation pipes was performed in July 2008 after a low power operation with the total beam power of $17 \mathrm{kWh}$. (The "total beam power" means a proton beam power integrated in time from the first beam on May 30, 2008.) In the measurement, we found an anomalous phenomenon that the dose rates around the pipes was almost unchanged or slightly increased after draining the mercury to the drain tanks. This means that our estimation on the hot-cell dose is much underestimated.

\section{Purpose of this study}

In this study, in order to figure out what is behind of the unexplained phenomenon, spectroscopy analysis for gamma rays from the mercury circulation system was carried out. Using the spectrum data, contribution rates of each radioactive product for total dose are deduced. In addition, for investigating measures for future hot-cell entry, the dose rate after the nominal operation with $1 \mathrm{MW}$ and the feature of dose disintegration by cooling are evaluated.

\section{Measurements}

The following three kinds of measurement data are obtained in the hot cell:

- Gamma-ray spectra before mercury drain

- Variation of gamma-ray spectra during mercury drain

- Absolute dose rate distribution.

Relation between the date of the data acquisition for each measurement and the proton beam power integrated from the first beam operation is shown in Fig. 2. Process and results of above three measurements are described in the following subsections.

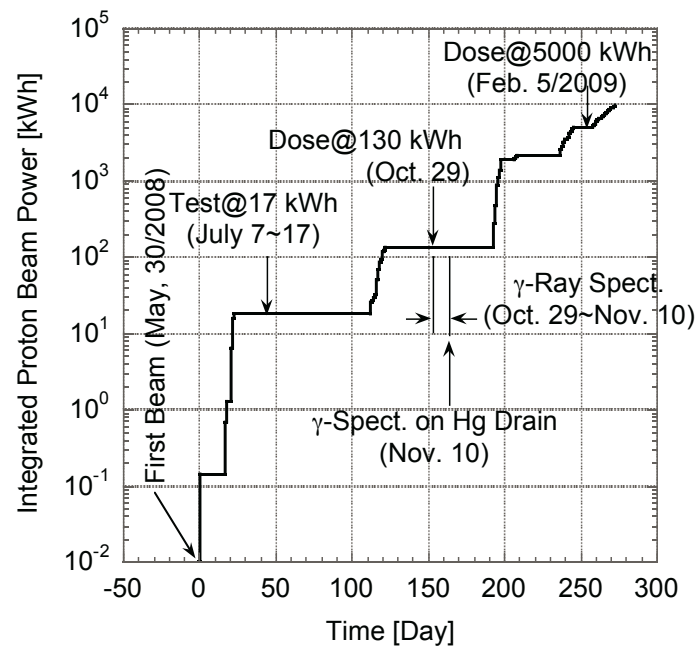

Fig. 2 Integrated proton beam power from the first beam operation of JSNS in 2008-2009.



Fig. 3 Photograph taken from the back of the target trolley in the hot cell. An HPGe detector with 30\% efficiency was located at a distance of $10 \mathrm{~m}$ from the back-end of the target trolley.

\section{Gamma-ray Spectroscopy}

Data acquisition of gamma-ray spectra in the hot cell started after the beam operation with the total beam power of $130 \mathrm{kWh}$ and 30-day cooling. An HPGe detector with 30\% efficiency was set at a distance of $10 \mathrm{~m}$ from the back of the trolley as shown in Fig. 3. In order to check the disintegration behavior of each gamma-ray peak, the spectrum data were obtained several times for 13 days.

The obtained gamma-ray spectrum at a cooling time of 30 days is shown in Fig. 4. Each gamma-ray peak was assigned by considering the energy and the half-life.

Using this data, the contribution rate of each nuclide assigned in the spectrum to total dose was deduced with a approximate method by using the following formula:

$$
R D^{(i)}=\frac{1}{N} \sum_{j} \frac{P^{(i)}\left(E^{(i)}{ }_{j}\right)}{E_{f}\left(E^{(i)}{ }_{j}\right)} C\left(E^{(i)}{ }_{j}\right) F\left(E^{(i)}{ }_{j}\right)
$$

where $R D^{(i)}$ is a contribution rate of $i^{\prime}$ th isotopes, $P^{(i)}\left(E^{(i)}\right)$ is a gamma-ray peak counts with an energy of $E^{(i)}$, which means energy of $j^{\prime}$ th gamma-ray for $i^{\prime}$ th isotope, $E_{f}\left(E^{(i)}\right)$ is a relative efficiency for parallel gamma beams at $E_{j}^{(i)}, C\left(E_{j}^{(i)}\right)$ is a flux-to-dose conversion factor, $F\left(E^{(i)}\right)$ is a correction factor for contribution of gamma-rays scattered inelastically at the concrete wall and other materials in the cell and $N$ is a normalization factor whose values are determined so that $\Sigma_{\mathrm{i}} R D^{(i)}=1$ for all assigned isotopes. The relative efficiency curve $E_{f}\left(E^{(i)}\right)$ for parallel gamma-rays was obtained by converting the efficiency curve measured at $20 \mathrm{~cm}$ from the detector surface in a manner of the point-detector concept ${ }^{3)}$. The correction factor of $F\left(E_{j}^{(i)}\right)$ was deduced by using the following expression:

$$
F\left(E_{j}^{(i)}\right)=\int_{E=0}^{E_{j}^{(i)}} \phi_{E_{j}^{(i)}}(E) C(E) d E / \int_{E=E_{j}^{(i)}-\Delta E / 2}^{E_{j}^{(i)}+\Delta E / 2} \phi_{E_{j}^{(i)}}(E) C(E) d E
$$

where $C(E)$ is a flux-to-dose conversion factor at $E$, and $\phi_{E(i) j}(E)$ is a gamma spectrum originated from the gamma-rays with $E^{(i)}{ }_{j}$ and $\Delta E \beta$ is a gamma-peak width. The 




Fig. 4 Gamma-ray spectrum at a distance of $10 \mathrm{~m}$ from the back-end of the target trolley in the hot cell. The spectrum was obtained at the cooling time of 30 days after beam operations with the total power of $130 \mathrm{kWh}$. Assigned isotopes are shown for each gamma peak. The symbol * shows ${ }^{188} \mathrm{Ir}$, and "BG" means "Natural Background". In the figure, the counts at 0-1000 and $1000-2000$ channels are multiplied by 10 and 100 , respectively.

gamma spectrum $\phi_{E(i) j}(E)$ at the position of the HPGe detector was evaluated using the Monte Carlo code of $\mathrm{MCNPX}^{4)}$ with use of the calculation model considered the concrete wall of the hot cell and the piping material. The factor of $F\left(E_{j}^{(i)}\right)$ ranged from 1.02 to 1.10 .

In the spectrum shown in Fig. 4, nine radioactive isotopes could be assigned, excluding natural background. The contribution rates for dose in the cell are tabulated in Table 1. As a result of the spectroscopy analysis, it was found that the radioactive isotope of ${ }^{188}$ Ir largely contributed to gamma dose in the cell and the contribution of ${ }^{203} \mathrm{Hg}$ was considerably small, contrary to our estimation. We also found that the gamma-ray peaks of ${ }^{188} \mathrm{Ir}$ decreased with a half-life about $10 \mathrm{~d}$ though the half-life of ${ }^{188} \mathrm{Ir}$ is $1.73 \mathrm{~d}$. This means that ${ }^{188} \mathrm{Ir}$ is produced by the decay of the mother nuclide, ${ }^{188} \mathrm{Pt}$, with a half-life of $10.2 \mathrm{~d}$.

\section{Variation during Mercury Drain}

After finishing the data acquisition of the gamma-ray spectra for 13 days, mercury in the circulation system was drained to the drain tanks. During the mercury drain, the
Table 1 Contribution rate $\left(R D^{(i)}\right)$ of each radioactive product for total dose rate at a distance of $10 \mathrm{~m}$ from the back-end of the target trolley

\begin{tabular}{||c|c|c||}
\hline \hline Isotope & Half-life & $\begin{array}{c}R D^{(i)} \text { :Contribution } \\
\text { Rate [\%] }\end{array}$ \\
\hline \hline${ }^{188} \mathrm{Ir}^{\text {a) }}$ & $1.73 \mathrm{~d}$ & 82.5 \\
\hline${ }^{185} \mathrm{Os}$ & $93.6 \mathrm{~d}$ & 5.4 \\
\hline${ }^{127} \mathrm{Xe}$ & $36.4 \mathrm{~d}$ & 3.4 \\
\hline${ }^{146} \mathrm{Eu}{ }^{\text {b) }}$ & $4.59 \mathrm{~d}$ & 3.0 \\
\hline${ }^{188} \mathrm{Pt}$ & $10.2 \mathrm{~d}$ & 2.0 \\
\hline${ }^{88} \mathrm{Y}$ & $106.7 \mathrm{~d}$ & 1.9 \\
\hline${ }^{203} \mathrm{Hg}$ & $46.6 \mathrm{~d}$ & 1.0 \\
\hline${ }^{175} \mathrm{Hf}$ & $70 \mathrm{~d}$ & 0.62 \\
\hline${ }^{189} \mathrm{Ir}$ & $13.2 \mathrm{~d}$ & 0.18 \\
\hline${ }^{18}$ It decays with a half-life of 10 days, which corresponds to
\end{tabular}

It decays with a half-life of 10 days, which corresponds to the half-life of the parent isotope: ${ }^{188} \mathrm{Pt}$.

b) It decays with a half-life of 50 days, which corresponds to the half-life of the parent isotope: ${ }^{146} \mathrm{Gd}$.

data of gamma-ray spectra were acquired. Time variations of counting rates for gamma-ray peaks of ${ }^{203} \mathrm{Hg},{ }^{185} \mathrm{Os}$ and ${ }^{188} \mathrm{Ir}$ were plotted in Fig. 5. While the counting rates of ${ }^{203} \mathrm{Hg}$ decreased drastically by draining as we expected, the others increased by a factor of 2 . This indicates that the some of the spallation products adhered on the inner surface of the piping wall and mercury in the circulation system works as shield before the drain. This means that mercury drain does not work for dose reduction in the hot cell.

\section{Measurement of Absolute Gamma Dose Rate}

Gamma dose distributions in the hot cell were measured with an ionization chamber after the operations of $130 \mathrm{kWh}$ (30-day cooling) and $5000 \mathrm{kWh}$ (5-day cooling). In Fig. 6, the distributions are plotted as a function of a distance from the back-end of the trolley.



Fig. 5 Time variation of gamma-ray peak counting rate during the mercury drain. The drain started at $15 \mathrm{~min}$ and finished at $40 \mathrm{~min}$. The counting rates of ${ }^{203} \mathrm{Hg}$ drastically decreased, but the others increased by a factor of 2 . This shows that the spallation products stuck on the inner surface of the circulation pipes. 




Fig. 6 Distributions of the dose rates in the hot cell. Measurements were carried out at cooling times of 30 days (after $130 \mathrm{kWh}$ operation) and 5 days (after $5000 \mathrm{kWh}$ operation).

\section{Future Prospect}

In order to summarize the measurement data for future use and discuss the measures for the hot-cell entry in future, time variation of the dose rate at $10 \mathrm{~m}$ after the nominal operation with $5000 \mathrm{MWh} / \mathrm{y} \quad(=1 \mathrm{MW} \times 5000 \mathrm{~h} / \mathrm{y})$ was evaluated as shown in Fig. 7. In the evaluation, on the basis of the measured data of gamma dose rates and contribution rates for major nuclide, history of the beam operation and half-lives of products were considered. The evaluation shows that the total dose rate at 10 -meter position after 10-day cooling is $5 \mathrm{mSv} / \mathrm{h}$, which is considerably larger than the evaluation ${ }^{2)}(=0.1 \mathrm{mSv} / \mathrm{h})$ by a factor of 50 .

Figure 7 shows that the total dose rate decreases with a half-life of 10 days at the beginning, but the disintegration speed slows down after 30 days due to ${ }^{185} \mathrm{Os}\left(\mathrm{T}_{1 / 2}=93.6 \mathrm{~d}\right)$ and ${ }^{175} \mathrm{Hf}\left(\mathrm{T}_{1 / 2}=70 \mathrm{~d}\right)$. In order to keep enough working time (more than 5 hours per day) in the hot cell, the dose rates need to be reduced to less than $100 \mu \mathrm{Sv} / \mathrm{h}$ since the administration dose limit of J-PARC is $500 \mu \mathrm{Sv}$ per day. It means that cooling time more than a year will be needed for working in the hot cell at the nominal operation, but that does not work by considering the operation schedule of MLF. One of the feasible measures is construction of an additional shield. The spectroscopy data will be useful for shield design on thickness, size, etc.

By comparing the results shown in Fig. 7 with calculation of gamma-dose rate from mercury using the point-source approximation ${ }^{2)}$, we found that the radioactive behavior for dominant isotopes such as ${ }^{188} \mathrm{Ir}$ and ${ }^{185} \mathrm{Os}$ are consistent each other. This means that the calculation of the radioactivity in mercury shown in Ref. 2 is almost valid. But, in order to evaluate the dose rate in the cell correctly, we should know the information of chemical behavior of spallation products in mercury.

\section{Conclusion}

The gamma dose distributions and the gamma-spectra data



Fig. 7 Dose rate variation expected after the operation with $5000 \mathrm{MWh}(=1 \mathrm{MW} \times 5000 \mathrm{~h})$.

behind the mercury circulation system in the hot cell were obtained. Analyzing the data, we found the followings:

- Some of the spallation products selectively adhere to the pipe walls in the mercury circulation system.

- The drain of mercury does not work for reducing the dose rate in the hot cell due to the adhered products.

- Additional shield is required for the hands-on maintenance work in the hot cell

\section{Acknowledgment}

The authors would like to appreciate to the Target Team of Neutron Source Section in MLF Division of J-PARC for their great help on the measurements in the hot cell. We are also grateful to Messrs. Takashi Nakazawa and Koichi Sato, who are members of Radiation Safety Section in J-PARC, for advises from the viewpoints of radiation safety. Drs. Fujio Maekawa and Masatoshi Futakawa gave us useful comments for this material.

\section{References}

1) Japan Proton Accelerator Research Complex (J-PARC), http://j-parc.jp/index-e.html.

2) T. Kai, Y. Kasugai, M. Harada, K. Haga, H. Kogawa and F. Maekawa, "Radioactivity estimation at J-PARC Spallation Neutron Source by Using the DCHAIN-SP 2001 and PHITS codes," Proc. 1st Workshop on Accelerator Radiation Induced Activation (ARIA '08), Paul Scherrer Institut, Switzerland, Oct. 13-17, 2008, P.182-187 (2009).

3) K. Kawade, H. Sakane, Y. Kasugai, M. Shibata, T. Iida, A. Takahashi and T. Fukahori, "Measurement Method of Activation Cross-Sections of Reactions Producing Short-Lived Nuclei with $14 \mathrm{MeV}$," Nucl. Instrum. Methods Phys. Res. A 496, 183 (2003).

4) L. S. Waters (Ed.), MCNPX ${ }^{\mathrm{TM}}$ User's Manual, LA-UR-02-2607, Los Alamos National Laboratory (LANL) (2002). 